JOURNAL OF

SYMPLECTIC GEOMETRY

Volume 12, Number 3, 585-597, 2014

\title{
SMOOTHINGS OF SINGULARITIES AND SYMPLECTIC SURGERY
}

\author{
Heesang Park And András I. Stipsicz
}

\begin{abstract}
Suppose that $C$ is a connected configuration of two-dimensional symplectic submanifolds in a symplectic 4-manifold with negative definite intersection graph $\Gamma_{C}$. Let $(S, 0)$ be a normal surface singularity with resolution graph $\Gamma_{C}$ and suppose that $W_{S}$ is a smoothing of $(S, 0)$. We show that if we replace an appropriate neighborhood of $C$ with $W_{S}$, then the resulting 4-manifold admits a symplectic structure. The operation generalizes the rational blow-down operation of Fintushel-Stern, and therefore our result extends Symington's theorem about symplectic rational blow-downs.
\end{abstract}

\section{Introduction}

Suppose that $X$ is a closed, oriented 4-manifold. Recall that in the rational blow-down procedure (introduced by Fintushel and Stern $[\mathbf{6}]$ and extended by Park [13]), the tubular neighborhood of a collection of embedded spheres $S=\left(S_{1}, \ldots, S_{k}\right)$ in $X$ is replaced by a specific compact 4 -manifold $W_{S}$ with boundary, providing the closed 4-manifold $X_{S}$. The spheres intersect each other according to a linear graph $\Gamma_{S}$, and their self-intersections are determined by the continued fraction coefficients of the ratio $-\frac{p^{2}}{p q-1}$ for some $p>q>0$ relatively prime integers. The 4 -manifold $W_{S}$ in the construction is a smoothing of the singularity with resolution graph $\Gamma_{S}$, specified by the property that it is a rational homology disk, i.e., $H_{*}\left(W_{S} ; \mathbb{Q}\right) \cong H_{*}\left(D^{4} ; \mathbb{Q}\right)$.

The success of the rational blow-down construction stems from the fact that it produces 4-manifolds with interesting differential topology: under favorable circumstances, there is a simple relation between the SeibergWitten invariants of the 4-manifold $X$ and the resulting 4-manifold $X_{S}[6]$. In specific cases, the nonvanishing of the Seiberg-Witten invariant of $X_{S}$ can be explained using symplectic topology: according to a result of Symington $[\mathbf{1 5}, \mathbf{1 6}]$, if $(X, \omega)$ is a symplectic 4-manifold and the spheres in the configuration $S$ are symplectic submanifolds (intersecting $\omega$-orthogonally), 
then $X_{S}$ admits a symplectic structure (hence by Taubes' theorem [17], it has nontrivial Seiberg-Witten invariants). This symplectic feature of the cut-and-paste construction has been extended to further configurations of symplectic surfaces in symplectic 4-manifolds and further smoothings of singularities in $[\mathbf{1}, \mathbf{7}-\mathbf{9}]$. The general case, however, remained open and was formulated as Conjecture 1.4 in [9]. The aim of the present paper is to prove this conjecture. Informally, the result says that if there is a connected configuration of symplectic surfaces in a symplectic 4-manifold which intersect each other according to a negative definite matrix, we collapse them to a point, and deform the resulting complex singularity, then the deformation "globalizes in the symplectic category."

To formulate the theorem precisely, suppose that $(X, \omega)$ is a closed symplectic 4-manifold and $C=\left(C_{1}, \ldots, C_{m}\right)$ is a collection of smooth, closed two-dimensional submanifolds which satisfy the following properties:

- each $C_{i}$ is a symplectic submanifold and $C=\cup C_{i}$ is connected,

- for $i \neq j, C_{i}$ intersects $C_{j} \omega$-orthogonally and

- the intersection matrix $I=\left(C_{i} \cdot C_{j}\right.$ ) (with the self-intersections in the diagonal) is negative definite.

Suppose that $\Gamma_{C}$ is the (connected) plumbing graph corresponding to the curve configuration $C$. By our assumption, it is a negative definite plumbing graph, where the vertex $v$ corresponding to the surface $C_{v}$ is decorated by the self-intersection $e_{v}=C_{v} \cdot C_{v}<0$ and by the genus $g_{v}=g\left(C_{v}\right) \geq 0$. According to a fundamental result of Grauert [11], for any such graph, there is a normal surface singularity $(S, 0)$ with resolution dual graph equal to $\Gamma_{C}$. (The analytic type of the singularity is not necessarily unique, although its topology is determined by the graph $\Gamma_{C}$.) Assume furthermore that $(S, 0)$ admits a smoothing with Milnor fiber $W_{C}$. (Not every surface singularity admits smoothing — our result below is meaningful only in case the singularity $(S, 0)$ is smoothable.)

The main result of the present paper then reads as follows.

Theorem 1.1. With the notations above, let $\nu C$ denote an $\omega$-convex tubular neighborhood of the union $\cup C_{i}$. Then, under the assumptions listed above, there is an orientation-reversing diffeomorphism $\phi: \partial(X-\operatorname{int} \nu C) \rightarrow \partial W_{C}$ such that the glued-up manifold

$$
X_{C}=(X-\operatorname{int} \nu C) \cup_{\phi} W_{C}
$$

admits a symplectic structure which is equal to the restriction of $\omega$ over $X-\nu C$. 
The main idea of the proof of the above result is the following: by Gay and Stipsicz [9], the configuration $C=\cup_{i} C_{i}$ admits an $\omega$-convex neighborhood $U_{C}$, with boundary $\partial U_{C}$ supporting a compatible contact structure $\xi_{C}$. The smoothing $W_{C}$, on the other hand, admits the structure of a Stein domain, inducing the so-called Milnor fillable contact structure $\xi_{M}$ on $\partial W_{C}$. By our assumption, $\partial U_{C}$ and $\partial W_{C}$ are orientation-preserving diffeomorphic 3-manifolds. The main tool in verifying Theorem 1.1 is the result showing that $\xi_{C}$ and $\xi_{M}$ are, in fact, contactomorphic. Therefore, taking an orientation-preserving contactomorphism $\psi:\left(\partial U_{C}, \xi_{C}\right) \rightarrow\left(\partial W_{C}, \xi_{M}\right)$, the gluing of symplectic 4-manifolds along contact hypersurfaces (as it is explained in [5], cf. also [12]) concludes the argument. In turn, the fact that the two contact structures $\xi_{C}$ and $\xi_{M}$ are contactomorphic will be proved by relating two compatible open book decompositions. The existence of this contactomorphism was verified in $[\mathbf{1 , 9}]$ for specific families of configurations of $C$; in this paper, we extend the result of [9] by constructing an appropriate horizontal open book decomposition on $\partial U_{C}$ compatible with $\xi_{C}$ (cf. Theorem 3.2). See also [2] for related results.

The paper is organized as follows. In Section 2, we quickly recall some facts about horizontal open book decompositions and in Section 3, we give the proof of the main result of the paper.

\section{Horizontal open book decompositions}

By the Giroux correspondence [10], open book decompositions play a central role in contact topology. For completeness, in this section we recall some facts and constructions regarding specific open book decompositions on plumbed 3 -manifolds. We start with a general definition.

Definition 2.1. Suppose that $Y$ is a given closed, oriented 3-manifold. The pair $(B, \varphi)$ is an open book decomposition on $Y$ if $B \subset Y$ is an oriented one-dimensional submanifold and $\varphi: Y-B \rightarrow S^{1}$ is a locally trivial fibration with the property that a fiber $\varphi^{-1}(t)$ is the interior of a Seifert surface of $B$. The submanifold $B$ is called the binding of the open book, while the closure of a fiber $\varphi^{-1}(t)$ is called a page. Two open book decompositions $(B, \varphi)$ and $\left(B^{\prime}, \varphi^{\prime}\right)$ of the diffeomorphic 3-manifolds $Y$ and $Y^{\prime}$ are equivalent if there is an orientation-preserving diffeomorphism $g: Y \rightarrow Y^{\prime}$ with the properties that $g(B)=B^{\prime}$ (as oriented 1-manifolds) and $\varphi=\varphi^{\prime} \circ g$.

According to the Giroux correspondence [10], an open book decomposition uniquely determines an isotopy class of compatible contact structures. Recall that the contact form $\alpha$ is compatible with the open book decompositions $(B, \varphi)$ if $B$ is tangent to the Reeb flow $R_{\alpha}$ defined by $\alpha$, while the interiors of the pages are transverse to $R_{\alpha}$. 
By a classical result of Stallings [14], in a rational homology 3-sphere an open book decomposition is determined by its binding. For manifolds with $b_{1}>0$, this principle no longer holds. By considering specific classes of 3-manifolds and open book decompositions, however, a similar statement can be proved. For the statement, we need a little preparation. (For related notions, see also [4].) Suppose that $Y$ is a graph manifold, i.e., it is given by the plumbing construction along a weighted graph $\Gamma$. This means that we consider circle bundles over the surfaces corresponding to the vertices (with Euler numbers specified by the framings of the graph) and plumb these pieces together.

Definition 2.2. An open book decomposition $(B, \varphi)$ on a graph manifold $Y$ is horizontal if the binding $B$ is the union of fibers of the individual fibrations, the pages are transverse to these fibrations and the orientation induced by the pages on the binding coincides with the orientation given by the fibration.

For a horizontal open book decomposition $(B, \varphi)$, let $\mathbf{n}=\left(n_{v}\right)$ denote the vector of nonnegative numbers specified by the binding components at each vertex $v$ of the plumbing graph $\Gamma$. Now the version of Stallings' result (due to Caubel-Némethi-Popescu-Pampu) is the following:

Theorem 2.3 (Proposition 4.6 of $[3]$ ). Suppose that $(B, \varphi)$ and $\left(B^{\prime}, \varphi^{\prime}\right)$ are two horizontal open book decompositions on the plumbing 3-manifold $Y=Y_{\Gamma}$. If $\mathbf{n}=\mathbf{n}^{\prime}$ and for each vertex $v$, we have $n_{v}=n_{v}^{\prime}>0$, then the two open book decompositions are equivalent, and hence the compatible contact structures are contactomorphic.

Suppose now that the plumbing graph $\Gamma$ of the plumbing 3 -manifold $Y_{\Gamma}$ is negative definite. In this case, $Y_{\Gamma}$ can be considered as the link of a (not necessarily unique) normal surface singularity. As such, it admits a Milnor fillable contact structure, which is independent from the chosen singularity [3]. According to a result of Caubel-Némethi-Popescu-Pampu, horizontal open book decompositions compatible with the Milnor fillable contact structure $\xi_{M}$ are easy to construct:

Proposition 2.4 (Theorem 4.1 of [3]). Let $p:(\widetilde{S}, E) \rightarrow(S, 0)$ be a good resolution of a normal surface singularity $(S, 0)$, where $E$ is a normal crossing divisor in $\widetilde{S}$ having smooth components $E_{1}, \ldots, E_{m}$ with $E=\sum_{i} E_{i}$. Assume that the nonzero effective divisor $D=\sum d_{i} E_{i}\left(d_{i} \in \mathbb{N}\right)$ satisfies

$$
\left(D+E+K_{\widetilde{S}}\right) \cdot E_{i}+2 \leq 0 \text { for any } i=1, \ldots, m
$$

Then, there exists a holomorphic function $f$ on $(S, 0)$ with an isolated singularity at 0 such that $\operatorname{div}(f \circ p)$ is a normal crossing divisor on $\widetilde{S}$ and 
the exceptional part of $\operatorname{div}(f \circ p)$ is D. Moreover, for each $i$, the number of intersection points $n_{i}=\operatorname{div}(f \circ p)_{s} \cdot E_{i}$ is strictly positive, where $\operatorname{div}(f \circ p)_{s}$ is the strict transform part of $\operatorname{div}(f \circ p)$.

By Caubel et al. [3, Remark 4.2], for any good resolution of the normal surface singularity $(S, 0)$, there is an effective divisor $D$ which satisfies the condition of Proposition 2.4. Consider now the open book decomposition determined by a function $f$ provided by Proposition 2.4: let $B=f^{-1}(0) \cap$ $\partial W_{C}$ and $\varphi=\frac{f}{|f|}$. As it was explained in [3, Example 4.5], the resulting open book decomposition is horizontal, compatible with $\xi_{M}$, and with the notation $n_{v}=-D \cdot E_{v}$ each $n_{v}$ is strictly positive.

Therefore, there are horizontal open book decompositions compatible with the Milnor fillable contact structure $\xi_{M}$, and indeed, we can find such open books with the extra condition that $n_{v}>0$ for all $v$. A useful simple observation shows that if $\mathbf{n}=\left(n_{v}\right)$ appears as such a vector, then so does $k \cdot \mathbf{n}$ for any positive integer $k$ :

Lemma 2.5. Let $D$ be an effective divisor which satisfies the conditions of Proposition 2.4. Then, any positive integer multiple $k \cdot D$ of $D$ also satisfies those conditions.

Proof. Let $D=\sum d_{i} E_{i}$ be an effective divisor satisfying $\left(D+E+K_{\widetilde{S}}\right) E_{i}+$ $2 \leq 0$ for all $i=1, \ldots, m$. Let $k$ be a positive integer. Then, $k((D+E+$ $\left.\left.K_{\widetilde{S}}\right) E_{i}+2\right) \leq 0$ for all $i$. By the adjunction equality $\sum_{j \neq i} E_{j} E_{i}+2 g\left(E_{i}\right)=$ $\left(E-E_{i}\right) \cdot E_{i}+\left(E_{i}+K_{\widetilde{S}}\right) \cdot E_{i}+2=\left(E+K_{\widetilde{S}}\right) E_{i}+2 \leq k\left(\left(E+K_{\widetilde{S}}\right) E_{i}+2\right)$ for all $i$. Furthermore, $0 \leq \sum_{j \neq i} E_{j} E_{i}+2 g\left(E_{i}\right)$ obviously holds, implying

$$
\left(k \cdot D+E+K_{\widetilde{S}}\right) E_{i}+2 \leq k\left(\left(D+E+K_{\widetilde{S}}\right) E_{i}+2\right) \leq 0
$$

for all $i$.

\section{Horizontal open book decompositions for $\xi_{C}$}

Now we turn our attention to constructing horizontal open book decompositions compatible with the contact structure $\xi_{C}$. First of all, following [9, Section 4], we extend the notion of an open book decomposition for manifolds with boundary as follows: if $M$ is a compact 3-manifold with nonempty boundary $\partial M$, then $(B, \varphi)$ is an open book decomposition if $B \subset M-\partial M$ is an oriented link and $\varphi: M-B \rightarrow S^{1}$ is a map which behaves near $B$ as a usual open book does and restricts to $\partial M$ as a fibration $\partial M \rightarrow S^{1}$.

Suppose that $Y$ is a plumbing 3-manifold along the plumbing graph $\Gamma$. To simplify notations, in the next two statements, we assume that two vertices of $\Gamma$ are connected by at most one edge. Let $v$ be a fixed vertex of the graph $\Gamma$ and suppose that $\left\{v_{1}, \ldots, v_{t_{v}}\right\}$ are the further vertices connected to $v$ in $\Gamma$. With $e_{v}$ denoting the framing fixed for $v$, let $N_{v}, N_{v_{j}}\left(\right.$ for $j=1, \ldots, t_{v}$ ) 
and $n_{v}$ be positive integers satisfying

$$
N_{v} e_{v}+\sum_{j=1}^{t_{v}} N_{v_{j}}=-n_{v} .
$$

In short, if $I$ denotes the intersection matrix of $\Gamma$ (with the $e_{v}$ 's in the diagonal), then $\mathbf{N} \cdot I=-\mathbf{n}$, where $\mathbf{N}=\left(N_{v}\right)$ and $\mathbf{n}=\left(n_{v}\right)$.

Let $D^{2}$ be a 2-disk containing disjoint small disks $D_{1}, \ldots, D_{t_{v}}$ and let $A=D^{2}-\cup_{i=j}^{t_{v}} \operatorname{int} D_{j}$. Consider $M=A \times S^{1}$ with coordinates $\beta \in S^{1}$ and $\gamma_{j} \in \partial D_{j}$ and $\alpha \in \partial D^{2}$ ( $\alpha$ and $\gamma_{j}$ with orientation as boundary of $D^{2}$ and $D_{j}$, respectively). Now an adaptation of $[\mathbf{9}$, Lemma 4.1] gives the following result.

Lemma 3.1. There exists an open book decomposition $(B, \varphi)$ on $M=A \times S^{1}$ such that the following conditions hold:

(1) $\left.\varphi\right|_{\partial D^{2} \times S^{1}}=-e_{v} N_{v} \alpha+N_{v} \beta$.

(2) $\left.\varphi\right|_{\partial D_{j} \times S^{1}}=N_{v_{j}} \gamma_{j}+N_{v} \beta$.

(3) The pages $\varphi^{-1}(\theta)$ are transverse to $\partial_{\beta}$.

(4) The binding $B$ is tangent to $\partial_{\beta}$.

(5) $B$ has $n_{v}$ components $B_{1}, \ldots, B_{n_{v}}$.

(6) When the pages are oriented so that $\partial_{\beta}$ is positively transverse, then $B_{1}, \ldots, B_{n_{v}}$ are oriented in the positive $\partial_{\beta}$ direction.

Proof. Let $p_{1}, \ldots, p_{n_{v}}$ be fixed points in $A$. We may assume that the centers of the disks $D_{1}, \ldots, D_{t_{v}}$ and the fixed points $p_{1}, \ldots, p_{n_{v}}$ lie on a line segment and that each $D_{j}$ and $p_{i}$ are contained in the interior of another disk $D_{j}^{\prime}$ for $1 \leq j \leq t_{v}+n_{v}$ such that the disks $D_{j}^{\prime}$ and $D_{j+1}^{\prime}$ are tangent to each other at one point and the center of $D_{j}^{\prime}$ is equal either to the center of $D_{j}$ or to $p_{j-t_{v}}$; see Figure 1 . The desired open book decomposition on $M=A \times S^{1}$ will be built from pieces.

First, we consider an index $j$ between 1 and $t_{v}$ and describe the open book decomposition on $\left(D_{j}^{\prime}-D_{j}\right) \times S^{1}$ over the annulus $D_{j}^{\prime}-D_{j}$. (Each such index $j$ corresponds to a vertex $v_{j}$ of the plumbing graph $\Gamma$ with the property that $v$ and $v_{j}$ are joined by an edge.) Now consider the curve $\left(N_{v},-N_{v_{j}}\right)$ on $\partial\left(\left(D_{j}^{\prime}-D_{j}\right) \times S^{1}\right)$. The boundary $\partial\left(\left(D_{j}^{\prime}-D_{j}\right) \times S^{1}\right)$ has two components, an inner and an outer one. In addition, the positive integers $N_{v}$ and $N_{v_{j}}$ are not necessarily relatively prime, therefore the resulting curve on one of the boundary components is not necessarily connected. Foliate the boundaries by these curves, and extend these foliations to a foliation of $\left(D_{j}^{\prime}-D_{j}\right) \times S^{1}$ by (possibly disjoint unions of) annuli (cf. the left-hand portion of Figure 1).

Consider now an index $j$ between $t_{v}+1$ and $t_{v}+n_{v}$. The disk $D_{j}^{\prime}$ then contains $p_{j-t_{v}}$. The open book decomposition on $D_{j}^{\prime} \times S^{1}$ will have binding equal to $\left\{p_{j-t_{v}}\right\} \times S^{1}$ and the pages provide a foliation of $\partial\left(D_{j}^{\prime} \times S^{1}\right)$ by 


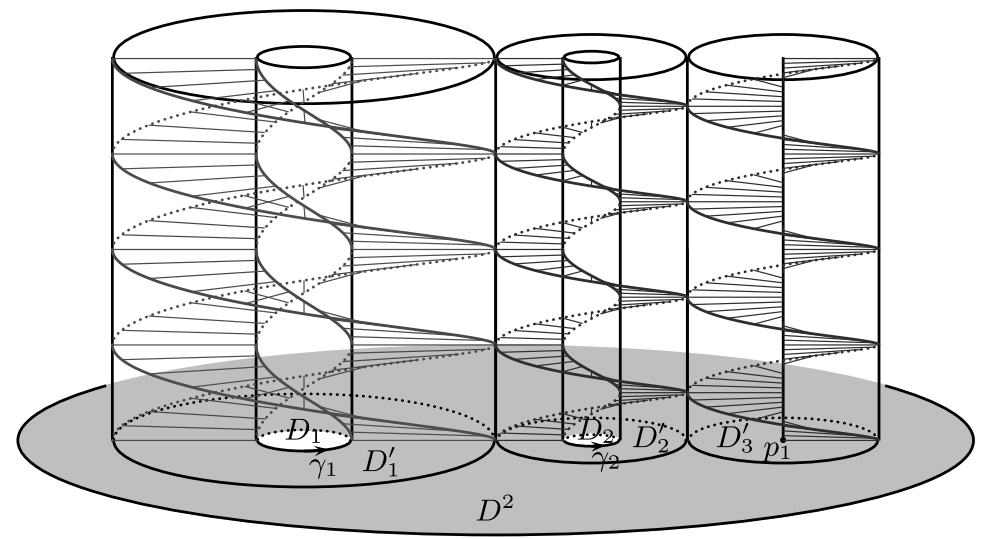

Figure 1. The diagram shows the special case when the vertex $v$ has two neighbors $v_{1}, v_{2}$, furthermore $N_{v}=4, N_{v_{1}}=$ $2, N_{v_{2}}=1$, and the value of $n_{v}$ is equal to 1 .

curves of type $\left(N_{v},-1\right)$. (These conditions uniquely determine the open book decomposition.) For an illustrative example, see the right-hand portion of Figure 1.

The union of the above pieces now provide an open book on $\left(\cup D_{j}^{\prime}\right) \times S^{1}$. After trivial smoothings over the tangencies of the consecutive disks, this construction extends to an open book decomposition on $A \times S^{1}$.

The proofs of Properties (2)-(6) are routine exercises inside the individual pieces. For verifying Property (1), we need to compute the slope of the fibration given by the pages on the outer boundary component $\partial D^{2} \times S^{1}$. By construction, the curves we get by intersecting the boundary with the pages are of type $\left(N_{v}, x\right)$, where $x$ is the sum of the corresponding coordinates on the individual pieces; cf. Figure 2. By our choices, we get that $x=$ $-n_{v}-\sum_{j} N_{v_{j}}$ where the summation goes for those vertices $v_{j}$ which are connected to $v$ in $\Gamma$. By our choice of the vector $\mathbf{N}$ (given in equation (3.1)), we get that $x=N_{v} e_{v}$, verifying Property (1).

According to a standard result (cf. [9, Corollary 3.4], for example), for a negative definite symmetric matrix $I$ with nonnegative off-diagonals, and for any $\mathbf{n} \in\left(\mathbb{R}^{+}\right)^{m}$, the vector $-\mathbf{n} \cdot I^{-1}$ is in $\left(\mathbb{R}^{+}\right)^{m}$. Once again, to simplify notation, in the following theorem assume that the graph $\Gamma$ contains no multiple edges.

Theorem 3.2. Suppose that $Y=Y_{\Gamma}$ is a given plumbing manifold, where $\Gamma$ is a negative definite plumbing graph on $m$ vertices with no multiple edges. Suppose that $\mathbf{n}=\left(n_{1}, \ldots, n_{m}\right)$ is a given vector in $\mathbb{N}^{m}$ with strictly positive entries. Then, there is a positive integer $k \in \mathbb{N}$ and a horizontal open book 


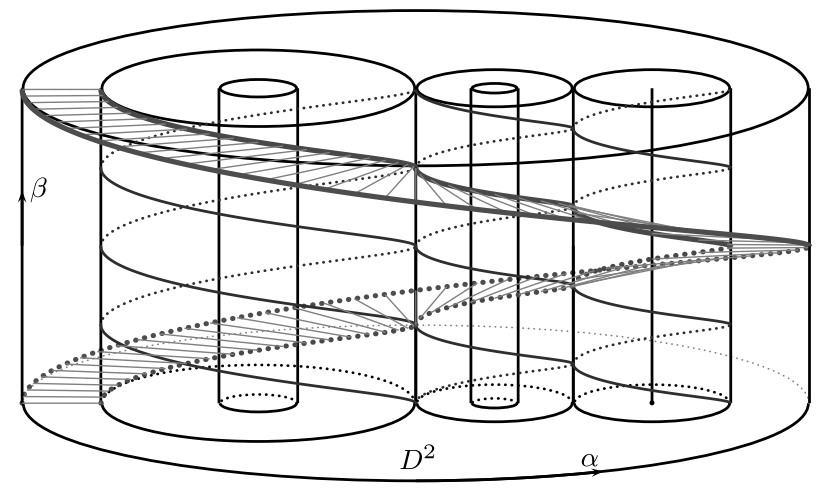

Figure 2. Computation of the slope of the curves on boundary of the union $\left(\cup D_{j}^{\prime}\right) \times S^{1}$. The diagram depicts part of the page we get by gluing the pages of the individual open book decompositions together.

decomposition $(B, \varphi)$ on $Y$ which is compatible with $\xi_{C}$ and at each vertex $v$ it has $k n_{v}$ binding components.

Proof. Let $\Gamma$ be the given negative definite plumbing graph with vertices corresponding to the surfaces $C_{v}(v=1, \ldots, m)$ with self-intersection numbers $C_{v}^{2}=e_{v}$ and genera $g\left(C_{v}\right)=g_{v}$. Our hypothesis on $\Gamma$ implies that $C_{v}$ intersects any other $C_{u}$ in at most one point in the plumbed 4-manifold $X_{\Gamma}$ with $\partial X_{\Gamma}=Y_{\Gamma}$.

For the given positive integral vector $\mathbf{n}$ consider $\mathbf{N} \in \mathbb{Q}^{m}$ satisfying the relation $\mathbf{N} \cdot I=-\mathbf{n}$ given by equation (3.1), where (as before) $I$ is the intersection matrix of the plumbing graph. The observation preceding Theorem 3.2 implies that $N_{v}$ is positive for all $v$. Notice that a priori each $N_{v}$ is a rational number, but after multiplying both sides of equation (3.1) by an appropriate positive integer $k$, we may assume that the resulting vector (which we still denote by $\mathbf{N}$ ) is integral. (We also keep the notation $\mathbf{n}$ for the vector after rescaling by $k$.)

For constructing a horizontal open book decomposition on $Y=Y_{\Gamma}$ with the required properties, we decompose the neighborhood of the union $C=$ $\cup C_{v}$ into fibered pieces: for the vertex $v$ of the graph $\Gamma$ we consider the $S^{1}$ bundle over $C_{v}$ with Euler number $e_{v}$. Let $\widehat{C}_{v}$ denote the punctured surface $C_{v}-D^{2}$. Suppose that $\partial \widehat{C}_{v} \times S^{1}$ has coordinates such that $\partial \widehat{C}_{v} \times\{1\}=m_{v}$, $\{p\} \times S^{1}=l_{v}$, and similarly $\partial D^{2} \times S^{1}$ has coordinates $\partial D^{2} \times\{1\}=\alpha_{v}$ and $\left\{p^{\prime}\right\} \times S^{1}=\beta_{v}$. We orient $\alpha_{v}$ by the boundary orientation of $D^{2}$ and $m_{v}$ with the orientation opposite to the boundary orientation of $\widehat{C}_{v}$. The $S^{1}$-bundle over $C_{v}$ with Euler number $e_{v}$ is given by gluing $D^{2} \times S^{1}$ and $\widehat{C}_{v} \times S^{1}$ with 
the gluing map induced by

$$
\alpha_{v}+e_{v} \beta_{v} \rightarrow m_{v} \quad \text { and } \quad \beta_{v} \rightarrow l_{v}
$$

The surface $C_{v}$ meets the union $\cup_{u \neq v} C_{u}$ in $t_{v}$ points. For each such intersection point (i.e., for each index $i$ between 1 and $t_{v}$ ) choose a small disk $D_{i} \subset D^{2}$. Let $A_{v}=D^{2}-\operatorname{int}\left(D_{1} \cup \cdots \cup D_{t_{v}}\right) \subset C_{v}$ be the complement of (the interiors) of the chosen disks. Near $C_{v}$, therefore, we can decompose the 3 -manifold as the union of $A_{v} \times S^{1}$ and $\widehat{C}_{v} \times S^{1}$. On $A_{v} \times S^{1}$, we take the horizontal open book decomposition provided by Lemma 3.1. On $\widehat{C}_{v} \times S^{1}$, we consider the horizontal foliation given by the map $\pi: \widehat{C}_{v} \times S^{1} \rightarrow S^{1}$ defined as $\pi=N_{v} l_{v}$. By Property (1) of Lemma 3.1, when we glue $A_{v} \times S^{1}$ and $\widehat{C}_{v} \times S^{1}$ via the gluing map specified by equation (3.2), the open book decompositions and the foliation glue together. Therefore, we have a horizontal open book decomposition near the surface $C_{v}$.

Let $q$ be an intersection point of $C_{v}$ and $C_{u}$ with $q \in D_{i_{1}} \subset C_{v}$ and $q \in D_{i_{2}} \subset C_{u}$. When we plumb the two bundles at $q$, we glue the circle bundles with the map $\gamma_{i_{1}} \rightarrow \beta_{i_{2}}$ and $\beta_{i_{1}} \rightarrow \gamma_{i_{2}}$ (where the curves $\gamma_{j}$ are as in Lemma 3.1). Thus, $N_{i_{2}} \gamma_{i_{1}}-N_{i_{1}} \beta_{i_{1}}$ maps to $-\left(N_{i_{1}} \gamma_{i_{2}}-N_{i_{2}} \beta_{i_{2}}\right)$. By Property (2) of Lemma 3.1, the curve $N_{i_{2}} \gamma_{i_{1}}-N_{i_{1}} \beta_{i_{1}}$ is part of the boundary of a page of the open book decomposition on $A_{v} \times S^{1}$. After plumbing, the pages of the open book decomposition are obtained by gluing pages of the open book decompositions on $A_{v} \times S^{1}$ and $A_{u} \times S^{1}$ along $N_{i_{2}} \gamma_{i_{1}}-N_{i_{1}} \beta_{i_{1}} \subset$ $\partial\left(A_{v} \times S^{1}\right)$ and $N_{i_{1}} \gamma_{i_{2}}-N_{i_{2}} \beta_{i_{2}} \subset \partial\left(A_{u} \times S^{1}\right)$. In conclusion, the pages of the individual open book decompositions glue together when performing the plumbing operation. This procedure, therefore, results an open book decomposition of $Y=Y_{\Gamma}$ with $n_{v}$ binding components near the surface $C_{v}$.

Finally, we need to check that this open book decomposition is compatible with the contact structure $\xi_{C}$. More precisely, we have to check that the Reeb vector field for a contact form representing $\xi_{C}$ is transverse to the pages and tangent to the binding components.

In order to identify the Reeb vector field of a contact structure representing $\xi_{C}$, we need to recall the construction of the $\omega$-convex neighborhood of $C=\cup_{v} C_{v}$ from [9, Section 3], where a 5-tuple $(U, \omega, C, f, V)$ is constructed with the following properties: $U$ is a tubular neighborhood of $C, \omega$ is a symplectic form on $U, f: U \rightarrow[0, \infty)$ is a smooth function with no critical values in $(0, \infty)$ and with $f^{-1}(0)=C$ and $V$ is a Liouville vector field on $U-C$ with $d f(V)>0$. Then, it easily follows that, for any small $t>0, f^{-1}[0, t]$ is an $\omega$-convex tubular neighborhood of $C$.

The construction of the 5 -tuple $(U, \omega, C, f, V)$ in $[\mathbf{9}]$ proceeds (similarly to the proof of Theorem 3.2) separately for the edges and the vertices of $\Gamma$ (and then the pieces are put together). For an edge $e$ connecting two vertices $v$ and $u$, the 5 -tuple $\left(U_{e}, \omega_{e},\left.C\right|_{e}, f_{e}, V_{e}\right)$ is given as follows: we choose a smooth 
function $g_{e}:[0, \infty) \times[0, \infty) \rightarrow[0, \infty)$ with properties given in $[\mathbf{9}$, p. 2211] (and contour shown by Gay and Stipsicz [9, Figure 1]). By appropriately chosen positive real numbers $z_{v}$ and $z_{u}$ corresponding to $v$ and $u$, and for a fixed $\delta>0$, we specify an open subset $R_{e}$ of $g_{e}^{-1}[0, \delta)$ with properties spelled out in $\left[\mathbf{9}\right.$, p. 2212], cf. [9, Figure 2]. Then, $\left(U_{e}, \omega_{e}\right)$ is defined by the unique connected symplectic 4-manifold with the toric moment map $\mu_{e}: U_{e} \rightarrow \mathbb{R}^{2}$ such that $\mu_{e}\left(U_{e}\right)=R_{e}$. Here, we can choose coordinates on $U_{e}$, with $p_{i} \in \mathbb{R}$ and $q_{i} \in \mathbb{R} / 2 \pi \mathbb{Z}$, such that $\omega_{e}=d p_{1} \wedge d q_{1}+d p_{2} \wedge d q_{2}$. Finally, we take $\left.C\right|_{e}=\mu_{e}^{-1}\left(\partial R_{e}\right), f_{e}=g_{e} \circ \mu_{e}$ and $V_{e}=p_{1} \partial_{p_{1}}+p_{2} \partial_{p_{2}}$.

The 5-tuple $\left(U_{v}, \omega_{v},\left.C\right|_{v}, f_{v}, V_{v}\right)$ corresponding to a vertex $v$ is given as follows: let $U_{v}=\left\{\left(\widehat{C}_{v} \cup A_{v}\right)-\partial\left(\widehat{C}_{v} \cup A_{v}\right)\right\} \times D_{\sqrt{2 \delta}}^{2}, \beta_{v}$ be a volume form on $C_{v}$ with the properties given in $[\mathbf{9}$, p. 2214] and let $(r, \theta)$ be polar coordinates on the disk $D_{\sqrt{2 \delta}}^{2}$. Then $\omega_{v}$ is defined by $\beta_{v}+r d r \wedge d \theta,\left.C\right|_{v}=\left\{\left(\widehat{C}_{v} \cup A_{v}\right)-\right.$ $\left.\partial\left(\widehat{C}_{v} \cup A_{v}\right)\right\}$ and $f_{v}=\frac{1}{2} r^{2}$. As in [9, p. 2214], we choose a specific Liouville vector field $W_{v}$ and define $V_{v}$ by $W_{v}+\left(\frac{1}{2} r+\frac{z_{v}}{r}\right) \partial_{r}$. The pieces then glue together to form the 5-tuple $(U, \omega, C, f, V)$ as it is described in [9, p. 2214].

In terms of the symplectic form $\omega$ and the Liouville vector field $V$, the Reeb vector field of the induced contact form can be calculated concretely: indeed, the vector field is a positive multiple of $\partial_{\theta}$ on $f_{v}^{-1}(t)$, and a positive multiple of $b_{1} \partial_{q_{1}}+b_{2} \partial_{q_{2}}$ for some $b_{1}, b_{2}>0$ on $f_{e}^{-1}(t)$. Since $\left\{q_{1}, q_{2}\right\}=$ $\left\{\gamma_{j}, \beta_{j}\right\}$ near $\partial D_{j}$, it follows that the open book decomposition constructed above is compatible with the contact structure $\xi_{C}$, concluding the proof of the theorem.

As a corollary of the arguments given above, now we can show that the two contact structures $\xi_{M}$ and $\xi_{C}$ are contactomorphic.

Corollary 3.3. Suppose that $C \subset(X, \omega)$ is a configuration of symplectic 2-manifolds as before, with $\omega$-convex neighborhood $U_{C}$ and induced contact structure $\xi_{C}$ on $\partial U_{C}$. Let $\xi_{M}$ be the Milnor fillable contact structure on the link of a singularity $(S, 0)$ with resolution graph $\Gamma_{C}$. Then, $\xi_{M}$ and $\xi_{C}$ are contactomorphic.

Proof. Assume first that the intersection graph $\Gamma_{C}$ of $C$ admits the further property that any two vertices are connected by at most one edge (and hence Theorem 3.2 applies).

Consider the resolution of the singularity $(S, 0)$ with dual graph $\Gamma_{C}$, and denote the irreducible components of the exceptional curve in the resolution by $\left\{E_{v}\right\}$. (These curves correspond to the vertices of the resolution graph $\Gamma_{C}$.) Let $D=\sum d_{i} E_{i}$ be an effective divisor satisfying the assumptions of Proposition 2.4. (By Caubel et al. [3, Remark 4.1] such D always exists.) As it is verified by Proposition 2.4, the existence of $D$ shows that there is a horizontal open book decomposition on $Y_{\Gamma_{C}}$ compatible with $\xi_{M}$ which has $n_{v}=-D \cdot E_{v}>0$ binding component at the vertex $v$. 
Define the vector $\mathbf{N}=\left(N_{v}\right)$ of positive rational numbers by the identity $\mathbf{N} \cdot I=-\mathbf{n}$, where $I$ is the intersection matrix of the plumbing graph $\Gamma_{C}$ and $\mathbf{N}=\left(N_{v}\right), \mathbf{n}=\left(n_{v}\right)$. Suppose that with the choice $k \in \mathbb{N}$ the products $k \cdot N_{v}$ are integers for all $v$. Consider the horizontal open book decomposition corresponding to the divisor $k \cdot D$. (As Lemma 2.5 shows, this divisor also satisfies the assumptions of Proposition 2.4.) This procedure provides a horizontal open book decomposition of $Y_{\Gamma_{C}}$ which is compatible with $\xi_{M}$ and has $k n_{v}>0$ binding components at each vertex $v$ of $\Gamma_{C}$.

Now apply Theorem 3.2 with the choice $\mathbf{n}$ and $k$ as above. As a result, we get a horizontal open book decomposition compatible with $\xi_{C}$ having $k n_{v}$ binding components at each vertex $v$. Therefore, the two contact structures $\xi_{M}$ and $\xi_{C}$ are compatible with horizontal open book decompositions with equal (and strictly positive) numbers of bindings at each vertex, hence by Theorem 2.3 the structures are contactomorphic.

Recall that at the beginning of the proof we assumed that $\Gamma_{C}$ has no multiple edges. In the general case, we repeatedly blow up the intersection points of the curves $C_{v}$ and $C_{u}$ until we get $\widetilde{C}_{v} \cdot \widetilde{C}_{u} \leq 1$ for the strict transforms of the curves in the original configurations. The newly introduced exceptional curves $F_{i}$ intersect other curves at most once, hence the new configuration $\widetilde{C}=\left(\widetilde{C}_{1}, \ldots, \widetilde{C}_{m}, F_{1}, \ldots, F_{t}\right)$ now satisfies the restriction under which we already proved the theorem. The blow-up procedure does not change the singularity $(S, 0)$ we have chosen for $\Gamma_{C}$, hence the Milnor fillable contact structures we get using $\Gamma_{C}$ or $\Gamma_{\widetilde{C}}$ are contactomorphic. Finally, let $\widetilde{\omega}$ be the symplectic form on the blow up of $(X, \omega)$, and let us consider an $\widetilde{\omega}$-convex neighborhood $U_{\widetilde{C}}$ of $\widetilde{C}$, defining the contact structure $\xi_{\widetilde{C}}$. Since by blowing down the newly introduced exceptional curves $F_{i}$ we get an $\omega$-convex neighborhood for $C$, we conclude that $\xi_{\widetilde{C}}$ and $\xi_{C}$ are contactomorphic. As a combination of the above said, we get that $\xi_{C}$ is contactomorphic to $\xi_{M}$ for any negative definite $\Gamma_{C}$, concluding the proof.

With these results at hand, now we can turn to the proof of the main result of the paper:

Proof of Theorem 1.1. Let $C=\left(C_{1}, \ldots, C_{m}\right)$ be the given configuration of symplectic surfaces in $(X, \omega)$, and $W_{C}$ a smoothing of a singularity $(S, 0)$ with resolution graph $\Gamma_{C}$ given by the configuration $C$. Let $U_{C}$ be an $\omega$-convex neighborhood of $C$ in $X$ (the existence of which is proved in $\left[\mathbf{9}\right.$, Theorem 1.2]). According to Corollary 3.3, the contact structure $\xi_{C}$ induced on $\partial U_{C}$ is contactomorphic to the Milnor fillable contact structure $\xi_{M}$ on $\partial W_{C}$. By the symplectic gluing theorem described in [5] (see also [12, Theorem 7.1.9]), for an orientation-preserving contactomorphism $\psi:\left(\partial U_{c}, \xi_{C}\right) \rightarrow\left(\partial W_{C}, \xi_{M}\right)$, we get an orientation-reversing diffeomorphism $\phi: \partial(X-\operatorname{int} \nu C) \rightarrow \partial W_{C}$ such that the manifold $X_{C}=\left(X-\operatorname{int} U_{C}\right) \cup_{\phi} W_{C}$ 
admits a symplectic structure $\omega_{C}$, which on $X-U_{C}$ coincides with the given symplectic structure $\omega$.

\section{References}

[1] A. Akhmedov and B. Ozbagci, Singularity links with exotic Stein fillings, J. Singul. 8 (2014), 39-49.

[2] M. Bhupal, Open book decompositions of links of simple surface singularities, Internat. J. Math. 20 (2009), 1527-1545.

[3] C. Caubel, A. Némethi and P. Popescu-Pampu, Milnor open books and Milnor fillable contact 3-manifolds, Topology 45 (2006), 673-689.

[4] T. Etgü and B. Ozbagci, Explicit horizontal open books on some plumbings, Internat. J. Math. 17 (2006), 1013-1031.

[5] J. Etnyre, Symplectic convexity in low-dimensional topology, in 'Symplectic, Contact and Low-Dimensional Topology' (Athens, GA, 1996) Topology Appl. 88, 3-25, 1998.

[6] R. Fintushel and R. Stern, Rational blowdowns of smooth 4-manifolds, J. Differential Geom. 46 (1997), 181-235.

[7] D. Gay and T. Mark, Convex plumbings and Lefschetz fibrations, J. Symplectic Geom. 11 (2013), 363-375.

[8] D. Gay and A. Stipsicz, Symplectic rational blow-down along Seifert fibered 3manifolds, Int. Math. Res. Not. IMRN 2007(22), 1-20.

[9] D. Gay and A. Stipsicz, Symplectic surgeries and normal surface singularities, Algebr. Geom. Topol. 9 (2009), 2203-2223.

[10] E. Giroux, Géométrie de contact: de la dimension trois vers les dimensions suprieures, in 'Proceedings of the International Congress of Mathematicians' (Beijing, 2002), Vol. II, Higher Education Press, Beijing, 2002, 405-414.

[11] H. Grauert, Über Modifikationen und exzeptionelle analytische Mengen, Math. Ann. 146 (1962), 498-507.

[12] B. Ozbagci and A. Stipsicz, Surgery on contact 3-manifolds and Stein surfaces, Bolyai Society Mathematical Studies, Vol. 13, Springer-Verlag, Berlin, János Bolyai Mathematical Society, Budapest, 2004.

[13] J. Park, Seiberg-Witten invariants of generalized rational blow-downs, Bull. Austral. Math. Soc. 56 (1997), 363-384.

[14] J. Stallings, Constructions of fibred knots and links, in 'Algebraic and Geometric Topology' (Proc. Symp. Pure Mathematics, Stanford University, Stanford, CA, 1976), Part 2, 55-60, Proc. Symp. Pure Mathematic, Vol. XXXII, Amer. Math. Soc., Providence, RI, 1978.

[15] M. Symington, Symplectic rational blowdowns, J. Differential Geom. 50 (1998), 505518.

[16] M. Symington, Generalized symplectic rational blowdowns, Algebr. Geom. Topol. 1 (2001), 503-518.

[17] C. Taubes, The Seiberg-Witten invariants and symplectic forms, Math. Res. Lett. 1 (1994), 809-822. 
Department of Mathematics

KONKUK UNIVERSITY

SEOUl 143-701, Korea

E-mail address: HeesangPark@konkuk.ac.kr

RÉNYi Institute of MATHEMatics

Budapest, Hungary

E-mail address: stipsicz.andras@math-inst.hu

Received 11/30/2012, accepted 03/18/2013

HP would like to thank András Némethi for valuable discussions. We would like to thank Burak Ozbagci and an anonymous referee for many helpful comments and suggestions. The main part of the work in this paper was done during HP's visit (supported by WPR program of KIAS) at the Alfréd Rényi Institute of Mathematics, whose hospitality and support are gratefully acknowledged. HP was supported by Basic Science Research Program through the National Research Foundation of Korea (NRF-2011-0012111). AS was supported by the Lendület project of the Hungarian Academy of Sciences and by ERC Grant LTDBud. 
